MELD best predicted mortality. The Rockall score was not a useful predictor of clinically important outcomes following AVH.

Competing interests None.

\section{PTH-095 خ OUTCOMES FOLLOWING ACUTE VARICEAL BLEEDING IN THE 2007 UK NATIONAL AUDIT}

doi:10.1136/gut.2011.239301.496

V Jairath, 1,* R Logan, ${ }^{2}$ S Hearnshaw, ${ }^{3}$ B Kahan, ${ }^{4}$ S Travis, ${ }^{5}$ M Murphy, ${ }^{1}$ K Palmer ${ }^{6}{ }^{1} \mathrm{NHS}$ Blood and Transplant, Oxford, UK: ${ }^{2}$ Division of Epidemiology and Public Health, Nottingham, UK; ${ }^{3}$ Royal Victoria Infirmary, Newcastle, UK; ${ }^{4} M R C$ Clinical Trials Unit, London, UK; ${ }^{5}$ John Radcliffe Hospital, Oxford, UK; ${ }^{6}$ Western General Infirmary, Edinburgh, UK

Introduction Most outcome data following acute variceal haemorrhage $(\mathrm{AVH})$ come from centres of expertise which may not reflect 'real-life' outcomes.

Methods To describe clinical characteristics and outcomes of patients presenting with AVH throughout the UK. The authors analysed data from the 2007 UK Comparative Audit of Upper Gastrointestinal Bleeding ${ }^{1}$. In this study prospective data were collected on consecutive patients presenting to 208 UK hospitals with all cause AUGIB between 1 May and 30 June 2007. Here the authors describe patients who presented with AVH. Results 599/6750 (8.9\%) presenting with AUGIB had known cirrhosis. $88 \%(526 / 599)$ of these presented with variceal bleeding and $12 \%$ (73/599) with non-variceal bleeding. $86 \%$ $(452 / 526)$ of those with AVH were acute admissions (median age 53 years; IOR 46-63, 68\% male). Before admission $8 \%$ $(43 / 526)$ were taking aspirin, 4\% (19/526) warfarin and $4 \%$ (22/526) NSAIDS. Median MELD score on admission was 15 (IOR 11-20). Prior to endoscopy 57\% (299/526) received an intravenous PPI, 44\% (232/526) vasopressin (or analogue), $27 \%$ $(144 / 526)$ antibiotics and $2 \%(11 / 526)$ tranexamic acid. $62 \%$ $(328 / 526)$ presented outside normal working hours $(\mathrm{OOH})$. For endoscopy, 30\% (157/526) were performed $\mathrm{OOH}$ and $66 \%$ $(346 / 526)$ within $24 \mathrm{~h}$ of presentation, with $12 \%(65 / 526)$ under general anaesthetic. $80 \%(418 / 526)$ had oesophageal varices and $11 \%(59 / 426)$ gastric varices as the source of bleeding, but just $64 \%(339 / 526)$ had a therapeutic procedure at the index endoscopy. 30\% (162/526) underwent a repeat endoscopy. $5 \%(30 / 526)$ had a Sengstaken-Blakemore tube inserted and $4 / 526(0.8 \%)$ patients underwent TIPSS. $26 \%(135 / 526)$ had rebleeding during admission and $15 \%(80 / 526)$ died. The median length of stay was 9 days (IOR 5-21 days). AUROC analysis showed that the admission MELD score usefully predicted mortality (AUROC 0.78), but poorly predicted the need for therapeutic endoscopy or rebleeding risk. The clinical Rockall score was a poor predictor of mortality, need for therapeutic intervention at endoscopy or rebleeding risk (AUROC $0.64,0.52$ and 0.61 respectively).

Conclusion Mortality following AVH may have improved in recent years, but notable deficiencies in the timeliness and use of endoscopic intervention for AVH persist. The admission 